\title{
Dynamic pricing problems with elastic demand
}

Citation for published version (APA):

Grigoriev, A., Hiller, B., Marban, S., Vredeveld, T., \& van der Zwaan, G. R. J. (2010). Dynamic pricing problems with elastic demand. METEOR, Maastricht University School of Business and Economics. METEOR Research Memorandum No. 053 https://doi.org/10.26481/umamet.2010053

Document status and date:

Published: 01/01/2010

DOI:

10.26481/umamet.2010053

Document Version:

Publisher's PDF, also known as Version of record

\section{Please check the document version of this publication:}

- A submitted manuscript is the version of the article upon submission and before peer-review. There can be important differences between the submitted version and the official published version of record.

People interested in the research are advised to contact the author for the final version of the publication, or visit the DOI to the publisher's website.

- The final author version and the galley proof are versions of the publication after peer review.

- The final published version features the final layout of the paper including the volume, issue and page numbers.

Link to publication

\footnotetext{
General rights rights.

- You may freely distribute the URL identifying the publication in the public portal. please follow below link for the End User Agreement:

www.umlib.nl/taverne-license

Take down policy

If you believe that this document breaches copyright please contact us at:

repository@maastrichtuniversity.nl

providing details and we will investigate your claim.
}

Copyright and moral rights for the publications made accessible in the public portal are retained by the authors and/or other copyright owners and it is a condition of accessing publications that users recognise and abide by the legal requirements associated with these

- Users may download and print one copy of any publication from the public portal for the purpose of private study or research.

- You may not further distribute the material or use it for any profit-making activity or commercial gain

If the publication is distributed under the terms of Article $25 \mathrm{fa}$ of the Dutch Copyright Act, indicated by the "Taverne" license above, 


\section{Maastricht University}

Alexander Grigoriev, Benjamin Hiller, Sebastián Marbán, Tjark Vredeveld, Ruben van der Zwaan

Dynamic Pricing Problems with Elastic Demand

$\mathrm{RM} / 10 / 053$

\section{METEOR}

Maastricht University School of Business and Economics 


\title{
Dynamic Pricing Problems with Elastic Demand
}

\author{
Alexander Grigoriev ${ }^{1} \quad$ Benjamin Hiller ${ }^{2} \quad$ Sebastián Marbán ${ }^{1}$ \\ Tjark Vredeveld ${ }^{1} \quad$ Ruben van der Zwaan ${ }^{1}$ \\ ${ }^{1}$ Department of Quantitative Economics \\ Maastricht University \\ P.O. Box 616, 6200 MD Maastricht, The Netherlands \\ Email: \{s.marban, a.grigoriev, t.vredeveld, r.vanderzwaan\}@maastrichtuniversity.nl \\ ${ }^{2}$ Department Optimization \\ Konrad-Zuse-Zentrum für Informationstechnik Berlin (ZIB) \\ Takustr. 7 D-14195 Berlin-Dahlem, Germany \\ Email: hiller@zib.de
}

\begin{abstract}
We study a dynamic pricing problem for a company that sells a single product to a group of customers over a finite time horizon. These customers are price sensitive and the price of today influences the group of customers of tomorrow. The objective is to set the prices over time so as to maximize revenue. We study two customer models: a multiplicative and an additive model.

Our main contribution is considering the case when the demand is deterministic. We give a polynomial time algorithm for the multiplicative model, and prove that the additive model is (weakly) NP-hard and allows a fully polynomial approximation scheme. Further, when the choice of prices is limited we prove that the optimal solution has a specific structure. Complementing the results for the deterministic setting, we finally provide two algorithms when the demand is stochastic.
\end{abstract}

\section{Introduction}

Dynamic pricing is an increasingly active field of research. An extensive overview of achievements in the field can be found in $[4,6,22]$. We consider a dynamic pricing problem for a company that sells a single product to a group of customers over a finite time horizon. These customers are price sensitive and the price of today influences the group of customers of tomorrow. The objective is to set the prices over time so as to maximize revenue. A deterministic, as well as a stochastic demand setting will be studied. We develop algorithms that run in polynomial time for several natural variants of the problem. Further, we show that one specific variant is NP-hard, present a pseudo-polynomial time algorithm, and transform this into a fully polynomial time approximation scheme.

Problem definition. We study the following dynamic pricing problem. For a finite time horizon of $T$ periods, a company needs to determine prices for a single product available in unlimited supply. Periods are indexed consecutively from $0, \ldots, T-1$. At each time period $t$, there are $C_{t}$ customers for the product and each of these customers buys a unit of the product only if the price, denoted by $\pi_{t}$, does not exceed the customer's reservation price. It is assumed that reservation prices are independent and identically distributed and that the probability distributions are given as part of the input. Let $F_{t}($.$) denote the cumulative probability distribution of the reservation$ 


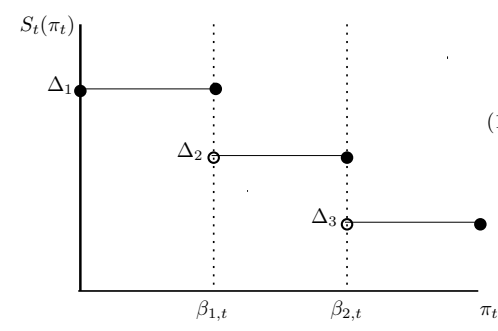

(a)

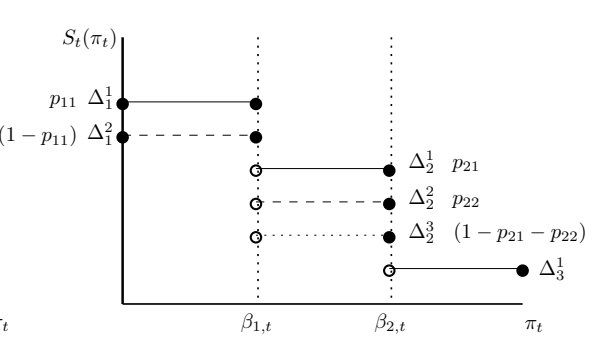

(b)

Figure 1: A deterministic and a randomized step function with two levels of change

price of a customer at time period $t$. The probability that a customer buys a unit of the product at time period $t$ is then given by $1-F_{t}\left(\pi_{t}\right)$.

The problem is to set the prices over the time horizon so as to maximize the total expected revenue. Formally,

$$
\max _{\pi}\{\mathbb{E}[R(\pi)]\}=\max _{\pi}\left\{\sum_{t} \pi_{t} C_{t}\left(1-F_{t}\left(\pi_{t}\right)\right)\right\},
$$

where $R(\pi): \mathbb{R}_{+}^{T} \rightarrow \mathbb{R}_{+}$denotes the total revenue given price vector $\pi=\left(\pi_{0}, \ldots, \pi_{T-1}\right)$.

We consider two customer models with elastic demand, in which the customers are price sensitive and the price of today influences the group of customers of tomorrow. Let $C_{0}$ be a given initial number of customers. In the first model, referred to as additive model, we assume that the number of customers at time $t$ changes by a price-dependent additive term

$$
C_{t}=C_{t-1}+S_{t-1}\left(\pi_{t-1}\right) \text {. }
$$

Here $S_{t}\left(\pi_{t}\right): \mathbb{R}_{+} \rightarrow\left\{\Delta_{1}, \ldots, \Delta_{k}\right\}$ with $\Delta_{i} \in\left\{-C_{0}, \ldots, C_{0}\right\}$ is a non-increasing step-function defined by $S_{t}\left(\pi_{t}\right)=\Delta_{i}$ if $\pi_{t} \in I_{i, t}$, where

$$
I_{i, t}= \begin{cases}{\left[0, \beta_{1, t}\right]} & \text { if } i=1, \\ \left(\beta_{k-1, t}, \infty\right) & \text { if } i=k, \\ \left(\beta_{i-1, t}, \beta_{i, t}\right] & \text { otherwise, }\end{cases}
$$

with given values $0=\beta_{0, t} \leq \ldots \leq \beta_{k, t}=\infty$. See Figure 1(a) for an illustration. By not allowing for price levels which would bring the total number of customers below zero, we enforce $C_{t} \geq 0$ for every $t \in\{0, \ldots, T\}$.

In the second model, referred to as multiplicative model, we assume that the number of customers at time $t$ changes by a multiplicative term

$$
C_{t}=\left(1+S_{t-1}\left(\pi_{t-1}\right)\right) C_{t-1},
$$

where $S_{t}\left(\pi_{t}\right)$ is defined as above, but with $\Delta_{1} \geq \ldots \geq \Delta_{k}>-1$. In what follows, we will refer to $\Delta_{i}$ as level of change $i$ in the number of customers and to $\beta_{i, t}$ as breakpoint $i$ of step function $S_{t}\left(\pi_{t}\right)$. Observe that the levels of change are the same for all time periods, whereas the breakpoints may change between time periods.

In addition to the deterministic demand setting, we also consider a stochastic demand setting in which the two customer models depend on a randomized step function. For each $i \in\{1, \ldots, k\}$, let $S_{t}^{i}\left(\pi_{t}\right)$ denote a random variable with discrete probability distribution, expressing the level of change if $\pi_{t} \in I_{i, t}$ at time $t$. The probability mass function of the distribution is given by

$$
\operatorname{Pr}\left[S_{t}^{i}\left(\pi_{t}\right)=\Delta_{i}^{j}\right]=p_{i j}
$$

with $0 \leq p_{i j} \leq 1$ for all $i \in\{1, \ldots, k\}$ and $j \in\left\{1, \ldots, \delta_{i}\right\}$, where $\delta_{i}$ indicates the number of sub-levels for each level of change $i$. Of course it must also hold that $\sum_{i=1}^{k} p_{i j}=1$ for all $j$. Further, $\Delta_{i}^{j}>-1$ 
in the multiplicative model and $\Delta_{i}^{j} \in\left\{-C_{0}, \ldots, C_{0}\right\}$ in the additive model. Figure 1(b) presents an illustration. Again, we enforce $C_{t} \geq 0$ for every $t$ by not allowing for price levels which could possibly lead to a negative customer level.

For every $i$, let $\pi_{i, t}^{*}$ denote the price $\pi_{t} \in I_{i, t}$ that maximizes $\pi_{t}\left(1-F_{t}\left(\pi_{t}\right)\right)$ : the expected revenue per customer at time $t$. We refer to this price as level price. For notational convenience, we write $\bar{\pi}_{i, t}^{*}=\pi_{i, t}^{*}\left(1-F_{t}\left(\pi_{i, t}^{*}\right)\right)$. The maximum expected revenue per customer at time period $t$ over all levels of change is then denoted by $\bar{\pi}_{\max , t}^{*}=\max \left\{\bar{\pi}_{1, t}^{*}, \ldots, \bar{\pi}_{k, t}^{*}\right\}$, and we define

$$
\bar{\pi}_{\max , C_{t}}^{*}=\max \left\{\bar{\pi}_{j, t}^{*} \mid j \in\{1, \ldots, k\} \text { s.t. } C_{t}+\Delta_{j} \geq 0\right\} .
$$

It is easy to see that an optimal solution to the dynamic pricing problem consists only of level prices. Furthermore, we assume that, for each $i$ and $t$, the maximum expected revenue per customer and the corresponding level price are returned by an oracle in constant time. For this reason, we omit the corresponding factor from the running time analysis, and count it as an elementary operation.

Previous work. Until a few decades ago, dynamic pricing methods were mainly considered to be a tool within the field of revenue management. The original literature of this field is build upon the pioneering work of $[2,3,15,20,21]$. Over the last few decades, dynamic pricing has received considerable attention from other research areas, like theoretical computer science, and its applications spread out to many other industries, see [4, 22]. According to [6], this is explained by swift developments in information technology, e-commerce, and Internet, which enabled companies to collect extensive amounts of customer data, and to use this to quickly update their prices if necessary, at almost no cost. Nowadays, dynamic pricing is an active field of research, $[8,14,19]$.

The problem studied in this paper is linked to the dynamic pricing field as follows. First, we focus on a discrete time model as in $[5,13]$, since companies in real life are unable to change their prices at all points in time, and therefore the continuous time model is impractical. Second, we study price-setting companies in line with [7] and [17], that is, companies that either have monopolistic market power, or that operate in a market with imperfect competition. Third, we consider a single perishable product with infinite capacity. The same product setting can be found in [16]. Finally, demand is determined by multiplying the group of potential customers by the probability that they purchase the product, similar to $[5,17,24]$. Customers are modeled as an autoregressive process, which constitutes a new approach in pricing problems. Models with autoregressive demand can also be found in related research fields, see e.g. [1, 11, 23], and [8]. The latter paper specifically states that prices tend to have lagged consequences.

In this paper, we investigate computational issues of dynamic pricing problems. Computational analysis is a well developed area in auction theory, for surveys see $[12,18]$. In the auction literature prices are usually determined by a bidding process. The focus of the present paper is, however, on dynamic pricing with posted prices. Here, depending on the prices customers can either decide to buy the product or abstain from buying. Pricing problems with posted prices are considered in the literature on Stackelberg games, see e.g. [9, 10]. All later papers consider the posted prices statically while this paper deals with pricing over time.

Our contribution. Our main contribution is the thorough exploration of the dynamic pricing problem with a deterministic additive model. We give a pseudo-polynomial time algorithm, and prove the complementary result that the problem is weakly NP-hard. Further, we present an fully polynomial time approximation scheme, which is a non-trivial transformation of the pseudo-polynomial time algorithm. Our non-algorithmic result is that the optimal solution has an interesting and nice structure in the case of stationary reservation prices and breakpoints. We complement the aforementioned results by a polynomial time algorithm when the number of breakpoints is constant.

We also explore the deterministic multiplicative model, for which we give a polynomial time algorithm. We conclude our paper by extending the polynomial time algorithm for the multiplicative model to the stochastic setting and developing a new algorithm for the stochastic additive model. 


\section{The deterministic additive model}

In this section, we study the dynamic pricing problem with a deterministic additive customer model.

\subsection{A pseudo-polynomial time dynamic programming algorithm}

Let $R_{t}\left(c_{t}\right)$ denote the maximum expected revenue obtained up to time period $t$, given $c_{t} \in \mathcal{C}_{t}$ customers, where $\mathcal{C}_{t}=\left\{\max \left\{0, C_{0}-t \Delta_{\max }\right\}, \ldots, C_{0}+t \Delta_{\max }\right\}$ with $\Delta_{\max }=\max \left\{\left|\Delta_{i}\right| \mid i \in\{1, \ldots, k\}\right\}$ for every $t$. The recursive formula for the update of the values $R_{t}\left(c_{t}\right)$ in the dynamic programming algorithm reads

$$
R_{t}\left(c_{t}\right)=\max \left\{R_{t-1}\left(c_{t}-\Delta_{u}\right)+\bar{\pi}_{u, t-1}^{*}\left(c_{t}-\Delta_{u}\right) \mid u \in\{1, \ldots, k\} \text { s.t. } c_{t}-\Delta_{u} \geq 0\right\}
$$

for time periods $t \in\{1, \ldots, T-1\}$ and $R_{0}\left(c_{0}\right)=0$. Then, the maximum expected revenue equals

$$
\max \left\{R_{T-1}\left(c_{T-1}\right)+\bar{\pi}_{\text {max }, c_{T}-1}^{*} c_{T-1} \mid c_{T-1} \in \mathcal{C}_{T-1}\right\} .
$$

This results in the following theorem.

Theorem 1. An optimal solution to the dynamic pricing problem can be found in $O\left(k T^{2} \Delta_{\max }\right)$ time.

Proof. We need to compute at most $2 T \Delta_{\max }$ values at time period $t$. Further, each of these values takes $O(k)$ time to obtain by expression (1). Hence, the total computation time is $O\left(k T^{2} \Delta_{\max }\right)$.

\subsection{NP-hardness}

In this subsection, we reduce the knapsack problem to the dynamic pricing problem with an additive model. Note that the knapsack problem has a polynomial time algorithm when all integers are bounded by a polynomial in the input length, as has our problem under consideration. Let an instance of knapsack be given by items $0, \ldots, n-1$ with weights $w_{0}, \ldots, w_{n-1}$, values $v_{0}, \ldots, v_{n-1}$ and capacity $W$. The objective is to find a subset of the items with the highest sum of values such that the sum of weights does not exceed the capacity. For notational ease, define $v_{\max }=$ $\max \left\{v_{i} \mid i \in\{0, \ldots, n-1\}\right\}$ and $w_{\max }=\max \left\{w_{i} \mid i \in\{0, \ldots, n-1\}\right\}$.

We construct an instance of the dynamic pricing problem from an instance of knapsack as follows. The number of time periods is set to $T=n$. Moreover, let $N \geq 6 n+\left\lceil\log v_{\max }\right\rceil+$ $\left\lceil\log w_{\max }\right\rceil$ be a large number, and define $C_{0}=L(n-1)+W$, where $L(i)=\sum_{j=0}^{i} 2^{j+2 N+n}$. For every time period $t \in\{0, \ldots, T-1\}$, we create for every item $i \in\{0, \ldots, n-1\}$ two levels of change $\Delta_{i}^{\text {pick }}=-2^{i+2 N+n}-w_{i}$ and $\Delta_{i}^{\text {discard }}=-2^{i+2 N+n}$ with corresponding level prices and breakpoints $\pi_{i}^{\text {pick }}=\beta_{i}^{\text {pick }}=2^{4 i+N}+\frac{v_{i} \cdot 2^{2 N}}{L(i)}$ and $\pi_{i}^{\text {discard }}=\beta_{i}^{\text {discard }}=2^{4 i+N}$. In addition, we create for every time period a zero price $\pi^{0}=0$ with $\Delta^{0}=\beta^{0}=0$, and a level of change $\Delta^{\infty}=-\infty$ corresponding to prices larger than $\pi_{n-1}^{\text {pick }}$. Lastly, we assume that customers buy a unit of the product at every price, i.e., $\left(1-F\left(\pi_{i}^{\text {pick }}\right)\right)=\left(1-F\left(\pi_{i}^{\text {discard }}\right)\right)=1$, for every $i$. It is easy to see that this instance can be constructed in polynomial time.

Intuitively, level prices $\pi_{i}^{\text {pick }}$ and $\pi_{i}^{\text {discard }}$ correspond to either picking item $i$ in the knapsack or discarding the item. We will show below that the way we set our levels of change and level prices forces an optimal solution to ask precisely one of the prices, $\pi_{i}^{\text {pick }}$ or $\pi_{i}^{\text {discard }}$, for every item $i$. First, we present two auxiliary results.

Lemma 1. Let $\left(\pi_{0}^{*}, \ldots, \pi_{T-1}^{*}\right)$ be an optimal solution to the dynamic pricing problem instance. Then, $\pi_{t}^{*} \geq \pi_{s}^{*}>0$ for all $t<s<T-1$ with $\pi_{v}^{*}=0$ for all $v \in\{t+1, \ldots, s-1\}$. 
Proof. In case a zero price is present in a solution to the dynamic pricing problem, it is clear that the total revenue is independent of the time at which the zero price is chosen. Therefore, zero prices can be moved to the end of the time horizon, thereby always satisfying the lemma. From this it follows that we can consider an $s$ such that $s=t+1$.

Next, suppose for a contradiction that $\pi_{t}^{*}<\pi_{t+1}^{*}$ for some $t$, where neither $\pi_{t}^{*}$ nor $\pi_{t+1}^{*}$ is equal to the zero price. Let $\Delta_{t}^{*}$ and $\Delta_{t+1}^{*}$ be the levels of change corresponding to the prices $\pi_{t}^{*}$ and $\pi_{t+1}^{*}$. Then, the total revenue obtained at times $t$ and $t+1$ is

$$
C_{t} \pi_{t}^{*}+\left(C_{t}+\Delta_{t}^{*}\right) \pi_{t+1}^{*}=C_{t} \pi_{t}^{*}+C_{t} \pi_{t+1}^{*}+\Delta_{t}^{*} \pi_{t+1}^{*} .
$$

By exchanging prices $\pi_{t}^{*}$ and $\pi_{t+1}^{*}$, we instead obtain a total revenue of

$$
C_{t} \pi_{t+1}^{*}+\left(C_{t}+\Delta_{t+1}^{*}\right) \pi_{t}^{*}=C_{t} \pi_{t+1}^{*}+C_{t} \pi_{t}^{*}+\Delta_{t+1}^{*} \pi_{t}^{*} .
$$

Note that the number of customers at time $t+2$ is not affected by the exchange.

By construction, we know that for some integers $0 \leq \tau_{1}<\tau_{2}$

$$
\begin{gathered}
2^{4 \tau_{1}+N} \leq \pi_{t}^{*} \leq 2^{4 \tau_{1}+N}+\frac{v_{\max } \cdot 2^{2 N}}{L\left(\tau_{1}\right)}, \\
-2^{\tau_{1}+2 N+n} \geq \Delta_{t}^{*} \geq-2^{\tau_{1}+2 N+n}-w_{\max },
\end{gathered}
$$

and

$$
\begin{gathered}
2^{4 \tau_{2}+N} \leq \pi_{t+1}^{*} \leq 2^{4 \tau_{2}+N}+\frac{v_{\max } \cdot 2^{2 N}}{L\left(\tau_{2}\right)}, \\
-2^{\tau_{2}+2 N+n} \geq \Delta_{t+1}^{*} \geq-2^{\tau_{2}+2 N+n}-w \max .
\end{gathered}
$$

Combining (3) and (4) gives an upper bound for $\Delta_{t}^{*} \pi_{t+1}^{*}$

$$
\Delta_{t}^{*} \pi_{t+1}^{*} \leq-2^{\tau_{1}+2 N+n} \cdot 2^{4 \tau_{2}+N}=-2^{\tau_{1}+4 \tau_{2}+3 N+n} .
$$

Further, a lower bound for $\Delta_{t+1}^{*} \pi_{t}^{*}$ is obtained by combining (2) and (5)

$$
\begin{aligned}
\Delta_{t+1}^{*} \pi_{t}^{*} & \geq\left(-2^{\tau_{2}+2 N+n}-w_{\max }\right)\left(2^{4 \tau_{1}+N}+\frac{v_{\max } \cdot 2^{2 N}}{L\left(\tau_{2}\right)}\right) \\
& >-2^{\tau_{2}+2 N+n+1}\left(2^{4 \tau_{1}+N}+\frac{v_{\max } \cdot 2^{2 N}}{L\left(\tau_{2}\right)}\right) \\
& >-2^{\tau_{2}+2 N+n+1}\left(2^{4 \tau_{1}+N}+1\right) \\
& =-2^{4 \tau_{1}+\tau_{2}+3 N+n+1}-2^{\tau_{2}+2 N+n+1} \\
& >-2^{4 \tau_{1}+\tau_{2}+3 N+n+2}
\end{aligned}
$$

where the third inequality follows from the definition of $L(i)$.

Finally, combining (6) and (7) brings us to

$$
\Delta_{t}^{*} \pi_{t+1}^{*} \leq-2^{\tau_{1}+4 \tau_{2}+3 N+n}<-2^{4 \tau_{1}+\tau_{2}+3 N+n+2} \leq \Delta_{t+1}^{*} \pi_{t}^{*}
$$

such that

$$
C_{t} \pi_{t}^{*}+C_{t} \pi_{t+1}^{*}+\Delta_{t}^{*} \pi_{t+1}^{*}<C_{t} \pi_{t+1}^{*}+C_{t} \pi_{t}^{*}+\Delta_{t+1}^{*} \pi_{t}^{*} .
$$

This contradicts the fact that $\left(\pi_{0}^{*}, \ldots, \pi_{T-1}^{*}\right)$ is optimal, and proves the lemma.

Lemma 2. Let $\left(\pi_{0}^{*}, \ldots, \pi_{T-1}^{*}\right)$ be an optimal solution to the dynamic pricing problem instance. Then, for all $t \in\{0, \ldots, T-2\}$ there does not exist an integer $\tau \in\{0, \ldots, T-2\}$ such that

$$
2^{4 \tau+N} \leq \pi_{t+1}^{*} \leq \pi_{t}^{*} \leq 2^{4 \tau+N}+\frac{v_{\max } \cdot 2^{2 N}}{L(\tau)} .
$$


Proof. Suppose for a contradiction that $2^{4 \tau+N} \leq \pi_{t+1}^{*} \leq \pi_{t}^{*} \leq 2^{4 \tau+N}+\frac{v_{\max } \cdot 2^{2 N}}{L(\tau)}$ for some $\tau \in$ $\{0, \ldots, T-2\}$. By construction, we know that for $s \in\{t, t+1\}$

$$
2^{4 \tau+N} \leq \pi_{s}^{*} \leq 2^{4 \tau+N}+\frac{v_{\max } \cdot 2^{2 N}}{L(\tau)}
$$

and

$$
-2^{\tau+2 N+n} \geq \Delta_{s}^{*} \geq-2^{\tau+2 N+n}-w_{\max } .
$$

Then, the total revenue obtained at times $t$ and $t+1$ is at most

$$
C_{t} \cdot 2^{4 \tau+N} \frac{v_{\max } \cdot 2^{2 N}}{L(\tau)}+\left(C_{t}+\Delta_{t}^{*}\right) 2^{4 \tau+N} \frac{v_{\max } \cdot 2^{2 N}}{L(\tau)}<2 C_{t} \cdot 2^{4 \tau+N} .
$$

However, the total revenue obtained by replacing the price at time $t$ by $\pi_{\tau+1}^{\text {discard }}$ and asking a zero price at time $t+1$ is at least

$$
C_{t} \cdot 2^{4(\tau+1)+N}=2 C_{t} \cdot 2^{4 \tau+N+3},
$$

which is strictly larger than $2 C_{t} \cdot 2^{4 \tau+N}$, the total revenue obtained before the replacement. In addition, the change in the number of customers is

$$
\Delta_{t}^{*}+\Delta_{t+1}^{*} \leq-2^{\tau+2 N+n} \cdot 2
$$

before the replacement, and $-2^{\tau+2 N+n+1}$ afterwards. That is, by replacing the prices we always end up with at least as many customers as before. This all contradicts the fact that $\left(\pi_{0}^{*}, \ldots, \pi_{T-1}^{*}\right)$ is optimal, and proves the lemma.

Lemma 3. Let $\left(\pi_{0}^{*}, \ldots, \pi_{T-1}^{*}\right)$ be an optimal solution to the dynamic pricing instance. Then, $\pi_{t}^{*}$ is equal to either $\pi_{n-t-1}^{\text {discard }}$ or $\pi_{n-t-1}^{\text {pick }}$ for all $t \in\{0, \ldots, T-1\}$.

Proof. It follows from Lemma 1 that prices in the optimal solution are monotone decreasing, therefore if $\pi_{i}^{\text {pick }}$ and $\pi_{i}^{\ell_{1}}$, with $\ell_{1} \in$ \{pick, discard\}, or two times $\pi_{i}^{\text {discard }}$ are asked, they are subsequent. However, Lemma 2 states that $\pi_{i}^{\ell_{1}}$ and $\pi_{i}^{\ell_{2}}$ with $\ell_{1}, \ell_{2} \in\{$ pick, discard $\}$ are never asked subsequently, for all $i \in\{0, \ldots, n-2\}$. Further, we cannot ask price $\pi_{n-1}^{\text {discard }}$ or $\pi_{n-1}^{\text {pick }}$ twice, nor any combination of the two, because $2 \cdot 2^{n-1+2 N+n}>C_{0}$. This proves the lemma in case there are no zero prices in the optimal solution. If zero prices are indeed asked, there exists an index $i$ such that neither $\pi_{i}^{\text {pick }}$ nor $\pi_{i}^{\text {discard }}$ is chosen. We will show below that this results in a contradiction.

Let us adjust the optimal solution by replacing any $\pi_{j}^{\text {pick }}$ by $\pi_{j}^{\text {discard }}$ for all possible $j$. Thereby, we will lose at most

$$
\begin{aligned}
n C_{0} \max \left\{\left(\pi_{l}^{\text {pick }}-\pi_{l}^{\text {discard }}\right) \mid l<n\right\} & =n(L(n-1)+W) \frac{v_{\max } \cdot 2^{2 N}}{L(0)} \\
& \leq n(L(n-1)+W)
\end{aligned}
$$

of the total revenue. Clearly, by construction there exists a feasible solution consisting of picking all discard prices.

Next, we ask price $\pi_{i}^{\text {discard }}$ instead of a zero price, and adjust the order of the prices asked in the optimal solution accordingly (Lemma 1). Then, the number of customers drops by $\left|\Delta_{i}^{\text {discard }}\right|$ for the remaining time steps, which results in a total loss of revenue of at most

$$
\left|\Delta_{i}^{\text {discard }}\right| \cdot \sum_{j=0}^{i-1} \pi_{j}^{\text {discard }}=2^{i+2 N+n} \cdot \sum_{j=0}^{i-1} 2^{4 j+N}<2^{i+2 N+n} \cdot 2^{4 i+N-3}=2^{5 i+3 N+n-3} .
$$


By the adjustments to the optimal solution proposed above, we further gain at least

$$
\begin{aligned}
\left(C_{0}+\sum_{j=i+1}^{n-1} \Delta_{i}^{\text {discard }}\right) \pi_{i}^{\text {discard }} & \geq\left(L(n-1)-\sum_{j=i+1}^{n-1} 2^{j+2 N+n}\right) \pi_{i}^{\text {discard }} \\
& =L(i) \cdot \pi_{i}^{\text {discard }}=L(i) \cdot 2^{4 i+N} \geq 2^{5 i+3 N+n}
\end{aligned}
$$

revenue.

Combining (8) and (9) and comparing to (10), we finally find that the gain of the adjustments is larger than the loss

$$
n \cdot(L(n-1)+W)+2^{5 i+3 N+n-3} \leq 2^{n} \cdot 2^{2 N+2 n}+2^{5 i+3 N+n-3}<2 \cdot 2^{5 i+3 N+n-3}<2^{5 i+3 N+n} .
$$

This is a contradiction to the fact that $\left(\pi_{0}^{*}, \ldots, \pi_{T-1}^{*}\right)$ is optimal. Hence, no zero prices are asked in an optimal solution, and at each moment of time $t$ we pick at most one price from $\left\{\pi_{n-t-1}^{\text {pick }}, \pi_{n-t-1}^{\text {discard }}\right\}$.

This brings us to the following theorem.

Theorem 2. The dynamic pricing problem with an additive model is NP-hard.

Proof. We construct a dynamic pricing instance from the knapsack instance as described in the beginning of the section. It is obvious by construction that every valid knapsack solution has a corresponding valid solution to the dynamic pricing problem. Furthermore, we show below that an optimal dynamic pricing solution has a corresponding optimal knapsack solution.

It follows from Lemma 3 that at time period $t$ we ask price $\pi_{n-t-1}^{\text {pick }}$ or $\pi_{n-t-1}^{\text {discard }}$. Let $x_{i}$ denote a binary variable indicating if price $\pi_{i}^{\text {pick }}$ or $\pi_{i}^{\text {discard }}$ is chosen for all $i \in\{0, \ldots, n-1\}$. That is, $x_{i}=1$ if we choose $\pi_{i}^{\text {pick }}$, and $x_{i}=0$ otherwise. Note that $\left(x_{n-1}, \ldots, x_{0}\right)$ is also a solution to the knapsack problem. Further, let $\rho_{n-t-1}=W-\sum_{j=n-t-1}^{n-1} x_{j} w_{j}$. Then, the number of customers at time period $t$ is

$$
\begin{aligned}
C_{t} & =C_{0}-\sum_{j=n-t-1}^{n-1} 2^{2 N+j+n}-\sum_{j=n-t-1}^{n-1} x_{j} w_{j} \\
& =L(n-t-1)+W-\sum_{j=n-t-1}^{n-1} x_{j} w_{j}=L(n-t-1)+\rho_{n-t-1},
\end{aligned}
$$

and the total revenue is given by

$$
\begin{aligned}
\mathbb{E}\left[R\left(\pi_{0}, \ldots, \pi_{T-1}\right)\right] & =\sum_{t=0}^{T-1} \pi_{T-t-1} C_{T-t-1} \\
& =\sum_{i=0}^{n-1}\left(x_{i}\left(2^{4 i+N}+\frac{v_{i} \cdot 2^{2 N}}{L(i)}\right)+\left(1-x_{i}\right)\left(2^{4 i+N}\right)\right) \cdot\left(L(i)+\rho_{i}\right) \\
& =\sum_{i=0}^{n-1} 2^{4 i+N} L(i)+\sum_{i=0}^{n-1} 2^{4 i+N} \rho_{i}+\sum_{i=0}^{n-1} x_{i} v_{i} 2^{2 N}+\sum_{i=0}^{n-1} x_{i} \frac{\rho_{i} \cdot v_{i} \cdot 2^{2 N}}{L(i)} .
\end{aligned}
$$

Intuitively, we are interested in $\sum_{i=1}^{n} x_{i} v_{i} 2^{2 N}$, which is the total value of the knapsack instance, shifted $2 N$ bits to the left. Note that $\sum_{i=1}^{n} 2^{4 i+N} L(i)$ is independent of the prices chosen such that we can subtract this from $\mathbb{E}\left[R\left(\pi_{0}, \ldots, \pi_{T-1}\right)\right]$ to obtain $\mathbb{E}\left[R^{\prime}\left(\pi_{0}, \ldots, \pi_{T-1}\right)\right]$. Furthermore, $\sum_{i=0}^{n-1} 2^{4 i+N} \rho_{i}$ and $\sum_{i=0}^{n-1} x_{i} \frac{\rho_{i} \cdot v_{i} \cdot 2^{2 N}}{L(i)}$ are dominated by $\sum_{i=0}^{n-1} x_{i} v_{i} 2^{2 N}$. This follows from the fact that the latter number is at least $2^{2 N}$ and the first two numbers are at most $n 2^{4 n+N}$ and $n$, respectively, with $N \geq 6 n+\left\lceil\log v_{\max }\right\rceil+\left\lceil\log w_{\max }\right\rceil$. By removing the first $2 N$ bits from the right of $\mathbb{E}\left[R^{\prime}\left(\pi_{0}, \ldots, \pi_{T-1}\right)\right]$, we obtain the total value of the knapsack instance. 


\subsection{A fully polynomial time approximation scheme}

In this subsection, we transform the pseudo-polynomial time dynamic programming algorithm of Section 2.1 into a fully polynomial time approximation scheme (FPTAS), that is, for any $\epsilon>0$, we have an algorithm that computes a solution with total expected revenue at least $(1-\epsilon)$ times the optimal total expected revenue, in time polynomial in the input and $1 / \epsilon$. First, we introduce some useful notation.

For a given solution $\pi=\left(\pi_{0}, \ldots, \pi_{T-1}\right)$, let $\eta_{i, t}(\pi)$ indicate the number of times level of change $\Delta_{i}$ is obtained up to time period $t$. Then, in the additive model the customer level at time period $t$ is given by

$$
C_{t}(\pi)=C_{0}+\sum_{s=0}^{t-1} S_{s}\left(\pi_{s}\right)=C_{0}+\sum_{i=1}^{k} \eta_{i, t}(\pi) \Delta_{i} .
$$

In case $\pi$ is clear from the context, we write $\eta_{i, t}$ and $C_{t}$.

Theorem 3. The dynamic pricing problem with an additive model admits an FPTAS.

Proof. Given $\epsilon>0$, we apply the dynamic programming algorithm of Section 2.1 to a rounded instance in which the initial customer level and each level of change $i$ is scaled by $\Lambda>0$ as follows: $\tilde{C}_{0}=\left\lfloor\frac{C_{0}}{\Lambda}\right\rfloor$ and $\tilde{\Delta}_{i}=\left\lfloor\frac{\Delta_{i}}{\Lambda}\right\rfloor . \Lambda$ will be defined later. Then, $\tilde{C}_{t}=\left\lfloor\frac{C_{0}}{\Lambda}\right\rfloor+\sum_{i=1}^{k} \eta_{i, t}\left\lfloor\frac{\Delta_{i}}{\Lambda}\right\rfloor$ is a customer level on the rounded instance.

Let $\pi=\left\{\pi_{i_{0}, 0}^{*}, \ldots, \pi_{i_{T-1}, T-1}^{*}\right\}$ be an optimal solution to the original instance $I$ and $\pi^{\prime}$ an optimal solution to the rounded instance $\tilde{I}$. We show that

$$
\mathbb{E}\left[R^{I}\left(\pi^{\prime}\right)\right] \geq \Lambda \mathbb{E}\left[R^{\tilde{I}}\left(\pi^{\prime}\right)\right] \geq \Lambda \mathbb{E}\left[R^{\tilde{I}}(\pi)\right] \geq(1-\epsilon) \mathbb{E}\left[R^{I}(\pi)\right],
$$

where $\mathbb{E}\left[R^{I}(\cdot)\right]$ and $\mathbb{E}\left[R^{\tilde{I}}(\cdot)\right]$ denote the total expected revenue on the original instance $I$ and the rounded instance $\tilde{I}$, respectively.

For the first inequality, we find

$$
\begin{aligned}
\mathbb{E}\left[R^{I}\left(\pi^{\prime}\right)\right] & =\sum_{t=0}^{T-1} \bar{\pi}_{t}^{\prime} C_{t}=\Lambda \sum_{t=0}^{T-1} \bar{\pi}_{t}^{\prime} \frac{C_{t}}{\Lambda}=\Lambda \sum_{t=0}^{T-1} \bar{\pi}_{t}^{\prime} \frac{C_{0}+\sum_{i=1}^{k} \eta_{i, t}\left(\pi^{\prime}\right) \Delta_{i}}{\Lambda} \\
& \geq \Lambda \sum_{t=0}^{T-1} \bar{\pi}_{t}^{\prime}\left(\left\lfloor\frac{C_{0}}{\Lambda}\right\rfloor+\sum_{i=1}^{k} \eta_{i, t}\left(\pi^{\prime}\right)\left\lfloor\frac{\Delta_{i}}{\Lambda}\right\rfloor\right)=\Lambda \mathbb{E}\left[R^{\tilde{I}}\left(\pi^{\prime}\right)\right],
\end{aligned}
$$

where $\bar{\pi}_{t}^{\prime}=\pi_{t}^{\prime}\left(1-F\left(\pi_{t}^{\prime}\right)\right)$. Further, the second inequality follows trivially from the optimality of solution $\pi^{\prime}$ on the rounded instance $\tilde{I}$.

To prove the third inequality, we have

$$
\begin{aligned}
\Lambda \mathbb{E}\left[R^{\tilde{I}}(\pi)\right] & =\sum_{t=0}^{T-1} \bar{\pi}_{i_{t}, t}^{*}\left(\Lambda \tilde{C}_{0}+\sum_{i=1}^{k} \eta_{i, t}(\pi) \Lambda \tilde{\Delta}_{i, t}\right) \\
& \geq \sum_{t=0}^{T-1} \bar{\pi}_{i_{t}, t}^{*}\left(C_{0}-\Lambda+\sum_{i=1}^{k} \eta_{i, t}(\pi)\left(\Delta_{i, t}-\Lambda\right)\right) \\
& =\mathbb{E}\left[R^{I}(\pi)\right]-\sum_{t=0}^{T-1} \bar{\pi}_{i_{t}, t}^{*} \Lambda(t+1) \\
& \geq \mathbb{E}\left[R^{I}(\pi)\right]-\sum_{t=0}^{T-1} \bar{\pi}_{\max , C_{t}}^{*} \Lambda(t+1) \\
& \geq \mathbb{E}\left[R^{I}(\pi)\right]-\Lambda T \max _{t \leq T-1}\left\{\bar{\pi}_{\max , C_{t}}^{*}(t+1)\right\} .
\end{aligned}
$$

Next, we distinguish between two cases in order to bound the number of customer levels at each time period $t$. Let $\Delta_{\max }^{+}=\max \left\{\Delta_{i} \mid i \in\{1, \ldots, k\}\right\}$ and $\Delta_{\max }^{-}=-\min \left\{\Delta_{i} \mid i \in\{1, \ldots, k\}\right\}$. 
First, assume that $\Delta_{\max }^{+} \geq \Delta_{\max }^{-}$or $C_{0} \leq T \Delta_{\max }^{+} \leq T \Delta_{\max }^{-}$and let $\Lambda=\frac{\epsilon \Delta_{\max }^{+}}{T}$. From (11), we then have

$$
\begin{aligned}
\Lambda \mathbb{E}\left[R^{\tilde{I}}(\pi)\right] & \geq \mathbb{E}\left[R^{I}(\pi)\right]-\Lambda T \max _{t \leq T-1}\left\{\bar{\pi}_{\max , C_{t}}^{*}(t+1)\right\} \\
& =\mathbb{E}\left[R^{I}(\pi)\right]-\epsilon \max _{t \leq T-1}\left\{\bar{\pi}_{\max , C_{t}}^{*}(t+1)\right\} \Delta_{\max }^{+} \\
& \geq \mathbb{E}\left[R^{I}(\pi)\right]-\epsilon \mathbb{E}\left[R^{I}(\pi)\right]=(1-\epsilon) \mathbb{E}\left[R^{I}(\pi)\right],
\end{aligned}
$$

where the last inequality follows from the lower bound

$$
\mathbb{E}\left[R^{I}(\pi)\right] \geq \max _{t \leq T-1}\left\{\bar{\pi}_{\max , t}^{*}\left(C_{0}+t \Delta_{\max }^{+}\right)\right\} \geq \max _{t \leq T-1}\left\{\bar{\pi}_{\max , t}^{*}(t+1)\right\} \Delta_{\max }^{+},
$$

which holds since $\left|\Delta_{i}\right| \leq C_{0}$ for all $i$, and from the fact that $\bar{\pi}_{\max , C_{t}}^{*} \leq \bar{\pi}_{\max , t}^{*}$ for all $t$.

In this first case, the number of customer levels is at most $2 T \Delta_{\max }^{+}$. Scaling each customer level by $\Lambda$ results in at most $2 T \tilde{\Delta}_{\max }^{+} \leq 2 T \frac{T}{\epsilon}$ customer levels. Hence, the computation time of the dynamic program on the rounded instance is polynomial in the input size and $\frac{1}{\epsilon}$, as required.

Second, assume that $\Delta_{\max }^{+}<\Delta_{\max }^{-}$and $C_{0}>T \Delta_{\max }^{+}$. Let $\Lambda=\frac{\epsilon C_{0}}{T^{2}}$. Then,

$$
\begin{aligned}
\Lambda \mathbb{E}\left[R^{\tilde{I}}(\pi)\right] & \geq \mathbb{E}\left[R^{I}(\pi)\right]-\Lambda T \max _{t \leq T-1}\left\{\bar{\pi}_{\max , C_{t}}^{*}(t+1)\right\} \\
& \geq \mathbb{E}\left[R^{I}(\pi)\right]-\Lambda T^{2} \max _{t \leq T-1}\left\{\bar{\pi}_{\max , C_{t}}^{*}\right\} \\
& =\mathbb{E}\left[R^{I}(\pi)\right]-\epsilon C_{0} \max _{t \leq T-1}\left\{\bar{\pi}_{\max , C_{t}}^{*}\right\} \\
& \geq \mathbb{E}\left[R^{I}(\pi)\right]-\epsilon \mathbb{E}\left[R^{I}(\pi)\right]=(1-\epsilon) \mathbb{E}\left[R^{I}(\pi)\right],
\end{aligned}
$$

where the last inequality follows from the trivial lower bound

$$
\mathbb{E}\left[R^{I}(\pi)\right] \geq \max _{t \leq T-1}\left\{\bar{\pi}_{\max , t}^{*}\left(C_{0}+t \Delta_{\max }^{+}\right)\right\} \geq \max _{t \leq T-1}\left\{C_{0} \bar{\pi}_{\max , t}^{*}\right\},
$$

and again from the fact that $\bar{\pi}_{\max , C_{t}}^{*} \leq \bar{\pi}_{\max , t}^{*}$ for all $t$.

In this second case, the number of customer levels is at most $2 C_{0}$. Scaling each customer level by $\Lambda$ results in at most $2 \tilde{C}_{0} \leq 2 \frac{T^{2}}{\epsilon}$ customer levels. Hence, also for this case the computation time of the dynamic program on the rounded instance is polynomial in the input size and $\frac{1}{\epsilon}$.

Note that despite its apparent simplicity, we need to distinguish between several different cases for the correctness of this FPTAS, resulting in two different lower bounds on the optimal expected total revenue on the original instance. Reason for this is the fact that the number of customer levels can differ between these cases and that each customer level has to be rounded to obtain a computation time that is polynomial in the input size and $\frac{1}{\epsilon}$.

\subsection{Structure of an optimal solution}

In this subsection, we investigate the structure of an optimal solution, assuming that the reservation price distributions and breakpoints stay constant over time, i.e., $F_{t}()=.F($.$) and \beta_{i, t}=\beta_{i}$ for all $i$ and $t$. In what follows, the time indices of the prices can thus be dropped. We start by observing that the prices of an optimal solution can be arranged in a specific order.

Lemma 4. Let $\pi_{j}^{*}$ be a level price. If there exists another level price, say $\pi_{i}^{*}$, with $\pi_{i}<\pi_{j}$ and $\bar{\pi}_{j}^{*} \leq \bar{\pi}_{i}^{*}$, then $\pi_{j}^{*}$ will never be chosen.

Proof. At every time period $t$, the expected revenue is given by $\pi_{t}\left(1-F\left(\pi_{t}\right)\right) C_{t}$. Since $\bar{\pi}_{j}^{*} \leq \bar{\pi}_{i}^{*}$, choosing $\pi_{t}=\pi_{i}^{*}$ instead of $\pi_{t}=\pi_{j}^{*}$ does not decrease the expected revenue at time period $t$. In addition, we have $\Delta_{i} \geq \Delta_{j}$ and thus $\pi_{t}=\pi_{i}^{*}$ also results in the same or a higher customer level at time period $t+1$. Hence, $\pi_{j}^{*}$ will never be chosen at time period $t$. 
We call the level prices that are never chosen recessive prices. Let $\Pi^{*} \subseteq\left\{\pi_{1}^{*}, \ldots, \pi_{k}^{*}\right\}$ denote the set all non-recessive prices. This brings us to an ordering $\succ$ of the level prices in $\Pi^{*}$ based on the following definition.

Definition 1. Let $\pi_{i}^{*}, \pi_{j}^{*} \in \Pi^{*}$. Then, the order $\succ$ is defined as follows: $\pi_{i}^{*} \succ \pi_{j}^{*}$ if and only if $\bar{\pi}_{j}^{*} \Delta_{i}>\bar{\pi}_{i}^{*} \Delta_{j}$.

Lemma 5. The relation $\succ$ is transitive: let $\pi_{i}^{*}, \pi_{j}^{*}, \pi_{k}^{*} \in \Pi^{*}$. If $\pi_{i}^{*} \succ \pi_{j}^{*}$ and $\pi_{j}^{*} \succ \pi_{k}^{*}$, then $\pi_{i}^{*} \succ \pi_{k}^{*}$.

Proof. Let $\pi_{i}^{*} \succ \pi_{j}^{*}$ and $\pi_{j}^{*} \succ \pi_{k}^{*}$. Further, assume that $\pi_{k}^{*} \succ \pi_{i}^{*}$, that is, $\bar{\pi}_{i}^{*} \Delta_{k}>\bar{\pi}_{k}^{*} \Delta_{i}$. Then, $\Delta_{k}>\bar{\pi}_{k}^{*} \Delta_{i} / \bar{\pi}_{i}^{*}$. Next, we have that $\bar{\pi}_{k}^{*} \Delta_{j}>\bar{\pi}_{j}^{*} \Delta_{k}$, so $\Delta_{k}<\bar{\pi}_{k}^{*} \Delta_{j} / \bar{\pi}_{j}^{*}$. Combining, we obtain $\bar{\pi}_{k}^{*} \Delta_{i} / \bar{\pi}_{i}^{*}<\bar{\pi}_{k}^{*} \Delta_{j} / \bar{\pi}_{j}^{*}$, which results in $\bar{\pi}_{j}^{*} \Delta_{i}<\bar{\pi}_{i}^{*} \Delta_{j}$. This contradicts the assumption that $\pi_{i}^{*} \succ \pi_{j}^{*}$, thereby proving the lemma.

In the theorem below, we show that an optimal pricing follows the above defined ordering.

Theorem 4. The level prices of an optimal solution to the dynamic pricing problem follow the ordering $\succ$ defined on $\Pi^{*}$.

Proof. Let $\pi_{i}^{*}, \pi_{j}^{*} \in \Pi^{*}$ be two level prices for which $\pi_{i}^{*} \succ \pi_{j}^{*}$. Further, let $\pi=\left(\pi_{0}, \ldots, \pi_{T-1}\right)$ and $\pi^{\prime}=\left(\pi_{0}^{\prime}, \ldots, \pi_{T-1}^{\prime}\right)$ denote two solutions with $\pi_{t^{\prime}}=\pi_{t^{\prime}+1}^{\prime}=\pi_{j}^{*}, \pi_{t^{\prime}+1}=\pi_{t^{\prime}}^{\prime}=\pi_{i}^{*}$ for a certain time period $t^{\prime}$, and $\pi_{s}=\pi_{s}^{\prime}$ for all $s \neq t^{\prime}, t^{\prime}+1$. Note that $C_{s}=C_{s}^{\prime}$ for all $s \neq t^{\prime}, t^{\prime}+1$. Then,

$$
\begin{aligned}
\mathbb{E}\left[R\left(\pi^{\prime}\right)\right]-\mathbb{E}[R(\pi)] & =\sum_{t} \bar{\pi}_{t}^{\prime} C_{t}^{\prime}-\sum_{t} \bar{\pi}_{t} C_{t} \\
& =\bar{\pi}_{t^{\prime}}^{\prime} C_{t^{\prime}}^{\prime}+\bar{\pi}_{t^{\prime}+1}^{\prime} C_{t^{\prime}+1}^{\prime}-\bar{\pi}_{t^{\prime}} C_{t^{\prime}}-\bar{\pi}_{t^{\prime}+1} C_{t^{\prime}+1} \\
& =\bar{\pi}_{i}^{*}\left(C_{t^{\prime}}^{\prime}-C_{t^{\prime}+1}\right)+\bar{\pi}_{j}^{*}\left(C_{t^{\prime}+1}^{\prime}-C_{t^{\prime}}\right) \\
& =\bar{\pi}_{i}^{*}\left(-\Delta_{j}\right)+\bar{\pi}_{j}^{*}\left(\Delta_{i}\right) \\
& =\bar{\pi}_{j}^{*} \Delta_{i}-\bar{\pi}_{i}^{*} \Delta_{j}>0,
\end{aligned}
$$

where the inequality follows from Definition 1 . Hence, $\mathbb{E}\left[R\left(\pi^{\prime}\right)\right]-\mathbb{E}[R(\pi)]>0$.

We remark that an optimal solution to the dynamic pricing problem can be found in $O\left(k T^{k}\right)$ time, by considering all different orderings. Further, an obvious consequence of Lemma 4 is that for every $\pi_{i}^{*}, \pi_{j}^{*} \in \Pi^{*}$ with $i<j$ and at least one of both corresponding levels of change positive, we have that

$$
\bar{\pi}_{j}^{*} \Delta_{i}>\bar{\pi}_{i}^{*} \Delta_{j} .
$$

Intuitively, Theorem 4 thus shows that over time we first choose prices such that the corresponding level of change is positive, i.e., we increase the number of customers. After this, we choose prices with smaller or even negative levels of change to increase the revenue.

\subsection{Low number of breakpoints}

In contrast to the previous section, let us assume that the reservation price distributions and breakpoints vary over time. For a given solution $\pi$, let $H_{t}(\pi)=\left(\eta_{1, t}(\pi), \ldots, \eta_{k, t}(\pi)\right)$ denote a $k$-tuple of $\eta_{i, t}(\pi)$ 's. Note that $\sum_{i=1}^{k} \eta_{i, t}(\pi)=t$. We write $H_{t}$ in case $\pi$ follows from the context. Further, we call a tuple $H_{t+1}=\left(\eta_{1, t+1}, \ldots, \eta_{k, t+1}\right)$ reachable from tuple $H_{t}=\left(\eta_{1, t}, \ldots, \eta_{k, t}\right)$ if $H_{t+1}$ differs from $H_{t}$ in exactly one element by one.

To obtain an optimal solution for the dynamic pricing problem, we model it as a longest path problem in a directed acyclic graph $D_{k}(V, A)$, hereafter referred to by $D_{k}$. The node set $V$ is as follows. For each non-negative customer level $C_{t}$ defined by a tuple $H_{t}$ at time period $t$, we have a node $v_{t, H_{t}}$. In addition, we have a sink node $v_{T, \emptyset}$ and we define node $v_{0, H_{0}}$ to be the source node. 
The arc set $A$ consists of $\operatorname{arcs}\left(v_{t, H_{t}}, v_{t+1, H_{t+1}}\right)$, where $H_{t+1}$ is reachable from $H_{t}$, and additional $\operatorname{arcs}$ connecting nodes $v_{T-1, H_{T-1}}$ to the sink node. Each arc $\left(v_{t, H_{t}}, v_{t+1, H_{t+1}}\right)$ has length

$$
l\left(v_{t, H_{t}}, v_{t+1, H_{t+1}}\right)=\bar{\pi}_{u, t}^{*} C_{t}\left(H_{t}\right),
$$

where $u$ denotes the index of the element in which $H_{t}$ and $H_{t+1}$ differ, and each of the arcs $\left(v_{T-1, H_{T-1}}, v_{T, \emptyset}\right)$ has length 0 .

It is easy to see that any solution with level prices to the dynamic pricing problem corresponds to a path in the graph $D_{k}$ with the same length and vice versa. We therefore complete this subsection by estimating the running time of the longest path computation in $D_{k}$.

Theorem 5. An optimal solution to the dynamic pricing problem can be found in $O\left(k T^{k}\right)$ time.

Proof. The number of distinct non-negative customer levels at time period $t$ is equal to $\left(\begin{array}{c}k+t-1 \\ t\end{array}\right) \in$ $O\left(t^{k-1}\right)$. By construction, we therefore have $|V| \in O\left(T^{k}\right)$ and $|A| \in O\left(k T^{k}\right)$. Next, the customer levels can be calculated in $O(k)$ time and the arc lengths in constant time. Hence, the directed acyclic graph $D_{k}$ is created in $O\left(k T^{k}\right)$ time. Further, it is well known that the longest path problem in $D_{k}$ can be solved in $O(|A|) \in O\left(k T^{k}\right)$ time. This brings us to a total time complexity of $O\left(k T^{k}\right)$.

\section{The deterministic multiplicative model}

In this section, we study the dynamic pricing problem with a deterministic multiplicative model. In order to solve the problem to optimality, we propose a dynamic programming algorithm based on the maximum expected revenue obtained between time periods $t$ and $T$ per customer present at time $t$. Let $R_{t}^{C}$ denote this value, i.e.,

$$
R_{t}^{C}=\max _{\pi}\left\{\frac{\sum_{s=t}^{T-1} C_{s} \pi_{s}\left(1-F_{s}\left(\pi_{s}\right)\right)}{C_{t}}\right\} .
$$

To calculate the values of $R_{t}^{C}$, note that for each customer at time $t$, there will be $\left(1+\Delta_{i}\right)$ customers at time $t+1$ when price $\pi_{i, t}^{*}$ is chosen. Therefore, we have the following recursive formula

$$
R_{t}^{C}=\max \left\{\bar{\pi}_{u, t}^{*}+\left(1+\Delta_{u}\right) R_{t+1}^{C} \mid u \in\{1, \ldots, k\}\right\}
$$

for time periods $t=T-1, \ldots, 1,0$, and $R_{T}^{C}=0$. Hence, the maximum expected revenue equals the maximum expected revenue per customer at time 0 multiplied by the initial number of customers, that is, $R_{0}^{C} \times C_{0}$.

Theorem 6. An optimal solution to the dynamic pricing problem can be found in $O(k T)$ time.

Proof. From the above considerations, we know that the dynamic programming algorithm computes an optimal solution. For the running time, notice that for each time period $t=T-1, \ldots, 1,0$ we need to compute a maximum of $k$ values. Each of these values takes constant time to obtain, and hence the total computation time is $O(k T)$.

\section{The stochastic customer models}

In this section, we adjust the constructed dynamic programming algorithms to solve the problem with a stochastic demand setting. Let $\eta_{i, t}^{j}(\pi)$ indicate the number of times each sub-level $\Delta_{i}^{j}$, $j \in\left\{1, \ldots, \delta_{i}\right\}$, is realized up to time period $t$. Notice that for each $i$ and $t \sum_{j=1}^{\delta_{i}} \eta_{i, t}^{j}=\eta_{i, t}$, and let $H_{i, t}(\pi)=\left(\eta_{i, t}^{1}(\pi), \ldots, \eta_{i, t}^{\delta_{i}}(\pi)\right)$ denote the tuple containing these values for each $i$ and $t$. Abusing notation, define $H_{t}(\pi)=\left(H_{1, t}(\pi), \ldots, H_{k, t}(\pi)\right)$ with $\sum_{i=1}^{k} \sum_{j=1}^{\delta_{i}} \eta_{i, t}^{j}(\pi)=t$. In case $\pi$ is known from the context, we will write $\eta_{i, t}, \eta_{i, t}^{\delta_{i}}, H_{i, t}$, and $H_{t}$. We call tuple $H_{t+1}$ reachable from tuple $H_{t}$ if $H_{t+1}$ differs in exactly one element from $H_{t}$. 


\subsection{Additive model}

In the additive model, we say that $C_{t}$ is a reachable customer level at time period $t$ if there exists a tuple $H_{t}$ such that

$$
C_{t}\left(H_{t}\right)=C_{0}+\sum_{i=1}^{k} \sum_{j=1}^{\delta_{i}} \eta_{i, t}^{j} \Delta_{i}^{j} .
$$

To each of reachable customer level at time period $t$, we assign in the stochastic setting a probability $p_{t}\left(H_{t}\right)$, defined by

$$
p_{t}\left(H_{t}\right)=\prod_{i=1}^{k}\left(\frac{\eta_{i, t} !}{\prod_{j=1}^{\delta_{i}} \eta_{i, t}^{j} !} \prod_{j=1}^{\delta_{i}} p_{i j}^{\eta_{i, t}^{j}}\right),
$$

which follows from the fact that given $\eta_{i, t}$ with $\eta_{i, t}^{j}$ realizations of level $\Delta_{i}^{j}$, the same customer level with probability $\prod_{j=1}^{\delta_{i}} p_{i j}^{\eta_{i, t}^{j}}$ can be obtained in $\frac{\eta_{i, t} !}{\prod_{j=1}^{\delta_{i}} \eta_{i, t}^{j} !}$ possible ways.

For the case with a low and fixed $k$ and $\delta_{\max }$, we develop an algorithm that finds the optimal solution in polynomial time. Let $R\left(H_{t}\right)$ denote the maximum total expected revenue up to time period $t$, given tuple $H_{t}$. Further, let $C_{t, k, H_{t}}$ denote the set of reachable customer levels $C_{t}\left(H_{t}\right)$ and let $P_{H_{t}}$ denote the set of corresponding probabilities. Then, the maximum expected revenue equals $\max _{H_{t}}\left\{R\left(H_{T}\right)\right\}$, where the values $R\left(H_{t}\right)$ for time periods $t \in\{1, \ldots, T-1\}$ are computed by

$$
R\left(H_{t}\right)=\max \left\{R\left(H_{t-1}\right)+\mathbb{E}\left[G_{u}\left(H_{t-1}\right)\right] \mid u \in\{1, \ldots, k\} \text { s.t. } \eta_{u, t} \geq 1\right\}
$$

and $R\left(H_{0}\right)=0$. Here, $\mathbb{E}\left[G_{u}\left(H_{t-1}\right)\right]$ denotes the expected gain obtained from choosing a price at time $t-1$ in between breakpoints $\beta_{u-1, t-1}$ and $\beta_{u, t-1}$, i.e.,

$$
\mathbb{E}\left[G_{u}\left(H_{t-1}\right)\right]=\sum_{j \in\left\{1, \ldots,\left|C_{t-1, k, H_{t-1}}\right|\right\}} \bar{\pi}_{u, t-1}^{*} c_{j} p_{j} .
$$

with $c_{j} \in C_{t-1, k, H_{t-1}}$ and $p_{j} \in P_{H_{t-1}}$. Note that $u$ is the index of the element in which $H_{t}$ and $H_{t-1}$ differ.

Theorem 7. An optimal solution to the dynamic pricing problem in a stochastic setting can be found in $O\left(T^{2\left(\delta_{\max } k-1\right)} k^{3} \delta_{\max }^{2}\right)$ time.

Proof. The number of distinct reachable customer levels at time period $t$ is at most $\left(\begin{array}{c}\delta_{\max } k+t-1 \\ t\end{array}\right)$ in both customer models. We therefore compute expression (12) over at most $O\left(T^{\delta_{\max } k-1}\right)$ tuples at time period $t$. The computation time of (12) is estimated by $k$ times the time to sum over at most $O\left(T^{\delta_{\max }{ }^{k-1}}\right)$ multiplications of prices, customer levels and corresponding probabilities. By the fact that the computation times of a customer level and of a probability are both $O\left(k \delta_{\max }\right)$, each multiplication can be done in $O\left(k^{2} \delta_{\max }^{2}\right)$ time. Combining all the above, we arrive at a total computation time of $O\left(T^{2\left(\delta_{\max } k-1\right)} k^{3} \delta_{\max }^{2}\right)$.

\subsection{Multiplicative model}

The stochastic dynamic pricing problem with a multiplicative model can be solved to optimality by adjusting the dynamic programming algorithm of Section 3. For every time period $t$, let $R_{t}^{C}$ again denote the maximum expected revenue from $t$ to $T$, divided by the number of customers. The recursive formula for the update of the values $R_{t}^{C}$ is then given by

$$
R_{t}^{C}=\max \left\{\bar{\pi}_{u, t}^{*}+\left(\sum_{j=1}^{\delta_{u}}\left(1+\Delta_{u}^{j}\right) p_{u j}\right) R_{t+1}^{C} \mid u \in\{1, \ldots, k\}\right\}
$$

for time periods $t \in\{0, \ldots, T-1\}$ and $R_{T}^{C}=0$. Hence, the maximum expected revenue equals $R_{0}^{C} \cdot C_{0}$, and the following theorem is obtained. 
Theorem 8. An optimal solution to the dynamic pricing problem in a stochastic setting can be found in $O\left(k T \delta_{\max }\right)$ time, where $\delta_{\max }=\max _{i}\left\{\delta_{i}\right\}$.

Proof. We need to compute at most $k$ values at time period $t$. Further, each of these values takes at most $\delta_{\max }$ times constant time to obtain. Hence, the total computation time is $O\left(k T \delta_{\max }\right)$.

\section{References}

[1] L.C. Alwan, J.J. Liu, and D. Yao. Stochastic characterization of upstream demand processes in a supply chain. IIE Transactions, 35(3):207-219, 2003.

[2] P. Belobaba. Airline yield management: an overview of seat inventory control. Transportation Science, 21(2):63-73, 1987.

[3] P. Belobaba. Application of a probabilistic decision model to airline seat inventory control. Operations Research, 37(2):183-197, 1989.

[4] G. Bitran and R. Caldentey. An overview of pricing models for revenue management. Manufacturing Service Operations Management, 5(3):203-229, 2002.

[5] G.R. Bitran and H.K. Wadhwa. Some structural properties of the seasonal product pricing problem. Working paper \#3897-96, M.I.T. Sloan School of Management, Cambridge, MA, USA, 1996.

[6] L.M.A. Chan, Z.J. Shen, D. Simchi-Levi, and J.L. Swann. Handbook of quantitative supply chain analysis: Modeling in the e-business era, chapter 9, pages 335-382. Kluwer Academic Publishers, Boston/Dordrecht/London, 2004.

[7] L.M.A. Chan, D. Simchi-Levi, and J. Swann. Pricing, production, and inventory policies for manufacturing with stochastic demand and discretionary sales. Manufacturing and Service Operations Management, 8(2):149-168, 2006.

[8] W. Elmaghraby and P. Keskinocak. Dynamic pricing in the presence of inventory considerations: Research overview, current practices, and future directions. Management Science, 49(10):1287 -1309, 2003.

[9] A. Grigoriev, J. Van Loon, M. Sviridenko, M. Uetz, and T. Vredeveld. Optimal bundle pricing with monotonicity constraint. Operations Research Letters, 36(5):609 -614, 2008.

[10] V. Guruswami, J.D. Hartline, A.R. Karlin, and D. Kempe. On profit-maximizing envy-free pricing. In SODA '05: Proceedings of the 16th Annual ACM-SIAM Symposium on Discrete Algorithms, pages 1164-1173, Philadelphia, PA, USA, 2005.

[11] T. Hosoda and S.M. Disney. The governing dynamics of supply chains: The impact of altruistic behaviour. Automatica, 42(8):1301 - 1309, 2006.

[12] P. Klemperer. Auction theory: a guide to the literature. Journal of Economic Surveys, 13(3):227-286, 1999.

[13] P.K. Kopalle, A.G. Rao, and J.L. Assuncao. Asymmetric reference price effects and dynamic pricing policies. Marketing Science, 15(1):60-85, 1996.

[14] Yuri Levin, Jeff McGill, and Mikhail Nediak. Dynamic pricing in the presence of strategic consumers and oligopolistic competition. Management Science, 55(1):32-46, 2009.

[15] K. Littlewood. Forecasting and control of passenger bookings. In AGIFORS: the 12th Annual Symposium Proceedings, pages 95-128, Nathanya, Israel, 1972. 
[16] M.S. Lobo and S. Boyd. Pricing and learning with uncertain demand. Working paper, Duke University, Durham, NC, USA, 2003.

[17] C. Maglaras and J. Meissner. Dynamic pricing strategies for multi-product revenue management problems. Manufacturing and Service Operations Management, 8(2):136-148, 2006.

[18] R.P. McAfee and J. McMillan. Auctions and bidding. Journal of Economic Literature, 25(2):699-738, 1987.

[19] Ioana Popescu and Yaozhong Wu. Dynamic pricing strategies with reference effects. Operations Research, 55(3):413-429, 2007.

[20] M. Rothstein. An airline overbooking model. Transportation Science, 5(2):180-192, 1971.

[21] M. Rothstein. Hotel overbooking as a markovian sequential decision process. Decision Sciences, 5(3):389-404, 1974.

[22] L.R. Weatherford and S. Bodily. A taxonomy and research overview of perishable-asset revenue management: Yield management, overbooking, and pricing. Operations Research, 40(5):831-844, 1992.

[23] X. Zhang. The impact of forecasting methods on the bullwhip effect. International Journal of Production Economics, 88(1):15-27, 2004.

[24] W. Zhao and Y.-S. Zheng. Optimal dynamic pricing for perishable assets with nonhomogeneous demand. Management Science, 46(3):375-388, 2000. 